\title{
Treatment of Obstructive Sleep Apnea Using Temporary Mandible Advancement Device: A Case Report
}

\author{
Young-Chan Choi, DDS, MS ${ }^{1}$, Jae-Sung Kwon, MD, MMedSci², Seong Taek Kim, DDS, MS, PhD ${ }^{1}$ \\ 'Department of Orofacial Pain and Oral Medicine, Dental Hospital, College of Dentistry, Yonsei University, Seoul, Korea \\ 2BK21 PLUS Project, Department and Research Institute of Dental Biomaterials and Bioengineering, College of Dentistry, Yonsei University, Seoul, Korea
}

Obstructive sleep apnea (OSA) is the most prevalent type of sleep apnea, and oral appliance may be one of the options for treatment of OSA. But, the problems with the oral appliance are high cost, possible low compliance, and complications such as temporomandibular disorder. In this article, we described a severe OSA case that was successfully improved by using temporary mandible advancement device, which was designed for therapeutic effect of mandible advancement with low cost and simplified fabrication procedure.

Sleep Med Res 2015;6(1):35-37

Key Words Obstructive sleep apnea, Mandible advancement device, Polysomnography.

Received: December 1, 2014

Revised: January 19, 2015

Accepted: March 2, 2015

Correspondence

Seong Taek Kim, DDS, MS, PhD Department of Orofacial Pain and

Oral Medicine, Dental Hospital,

College of Dentistry, Yonsei University,

50 Yonsei-ro, Seodaemun-gu, Seoul

$120-752$, Korea

Tel $+82-2-2228-3110$

Fax +82-2-393-5673

E-mail k8756050@yuhs.ac

\section{INTRODUCTION}

Obstructive sleep apnea (OSA) is the most prevalent type of sleep apnea that is characterized by the predominant recurrence of obstructive events, resulting from a partial or complete collapse of the upper airway during sleep.

Risk factors for OSA include age, male sex, familial history, excess body fat, central obesity, large neck circumference, and craniofacial \& upper airway abnormalities like micrognathia, macroglossia, and tonsillar hypertrophy. ${ }^{1}$

The oral appliance (OA) may be one of the therapeutic options for the OSA. According to the treatment guideline, ${ }^{2}$ its use is recommended in the cases of patients who present with primary snoring, mild or moderate sleep apnea, continuous positive airway pressure (CPAP) intolerance, and failed surgical interventions as a substitutive therapy. Also, because the advancement of tongue or mandible by OA would increase the diameter of the upper airway tract, it seems to be a good indication for OSA.

However, there are some problems for the treatment of OSA with OA. There are a small number of dentists who are specifically trained to deal with sleep disorders, and the cost for OA is high. Even if the treatment with OA is initiated, it may be stopped due to jaw discomfort, occlusal change, or temporomandibular disorder. ${ }^{3}$ Therefore, there is a need for the development of an appliance that can be maneuvered easily, with low cost, which can also indicate whether improvements can be made through mandible advancement by wearing the device during sleep.

In this article, we described an OSA case that was successfully improved by using a temporary mandible advancement device, which was designed for the therapeutic effect of mandible advancement with low cost and simplified fabrication procedure at chair-side.

\section{CASE REPORT}

A 57-year-old man was presented at Department of Orofacial Pain and Oral Medicine at 

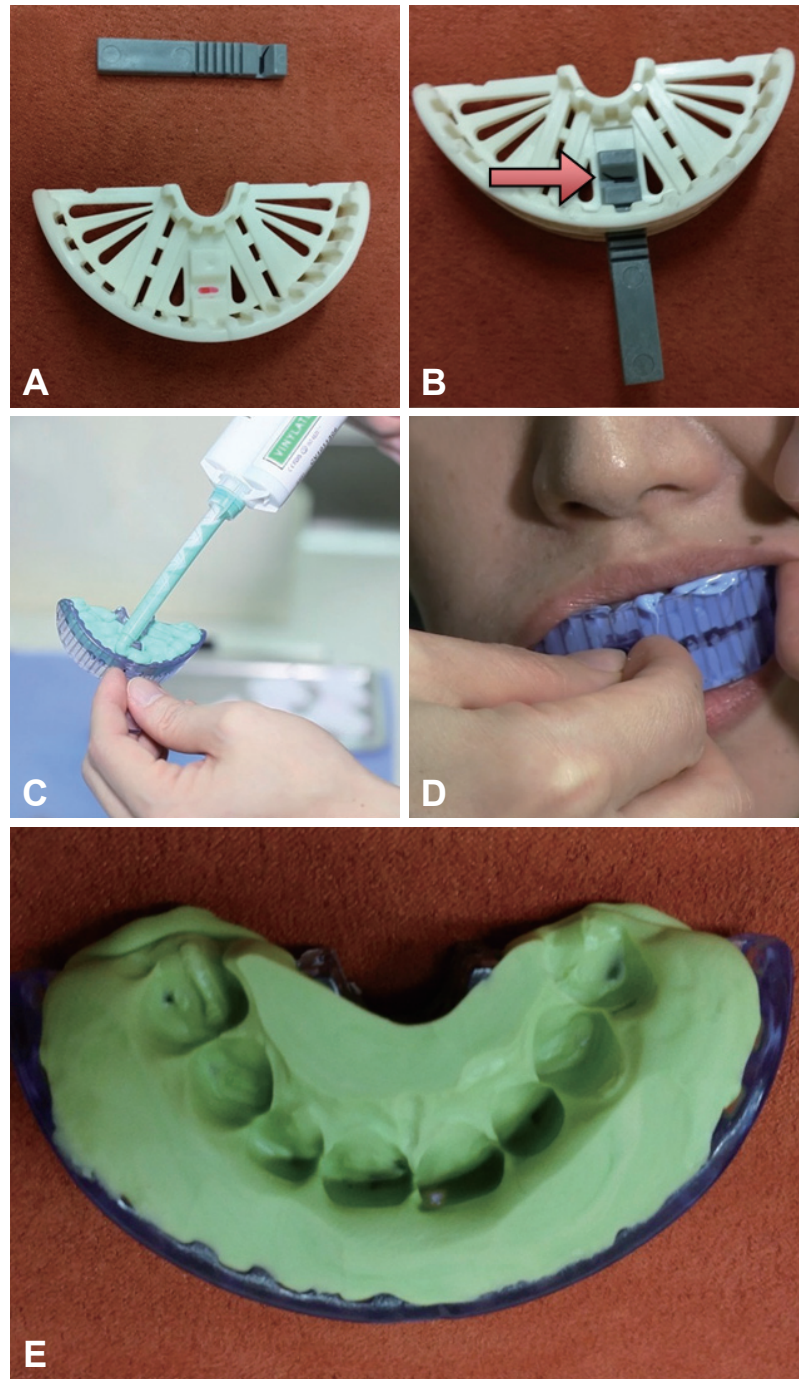

Fig. 1. Fabrication procedures of oral appliance. A: Tray part and guide pin were prepared. B: Guide pin was connected to the tray and adjusted for the mandible advancement, while patient's incisor was placed on the pre-engraved position (red arrow). C: Polyvinylsiloxane impression material was filled into the tray. D: The tray was then placed into inter-incisal space of patient, and the patient placed a bite on the tray. E: The tray was trimmed and finished into no-snore tray appliance. The guide pin was removed.

Dental Hospital of Yonsei University College of Dentistry, with the complaint of snoring accompanied by apnea during sleep and daytime sleepiness with polysomnography (PSG) result from Local Clinic. His Epworth Sleepiness Scale score was higher than 19 (the normal value is $<10$ ), and his body mass index was $27.5 \mathrm{~kg} / \mathrm{m}^{2}$.

According to the results of PSG, the apnea/hypopnea index (AHI) was 27.4 per hour of sleep, respiratory disturbance index (RDI) was 45.8 , and sleep efficiency was $77.8 \%$. Total arousal period, non-rapid eye movement (REM) sleep, and REM sleep accounted for $20.9 \%, 73.1 \%$, and $4.7 \%$ of the sleep duration, respectively.

Patient showed low proportion of deep stage sleep and REM
Table 1. PSG result before and after use of oral appliance

\begin{tabular}{lcc}
\hline & Before & After \\
\hline Sleep efficiency (\%) & 77.8 & 83.5 \\
Total arousal period (\%) & 20.9 & 16.5 \\
Non-REM sleep (\%) & 73.1 & 63.4 \\
N1 (\%) & 26.2 & 15.5 \\
N2 (\%) & 46.9 & 47.9 \\
REM sleep (\%) & 4.7 & 20.1 \\
AHI & 27.4 & 2.4 \\
RDI & 45.8 & 5.9 \\
Minimal $\mathrm{O}_{2}$ saturation (\%) & 79.0 & 88.0 \\
\hline
\end{tabular}

PSG: polysomnography, REM: rapid eye movement, AHI: apnea/ hypopnea index, RDI: respiratory disturbance index.

sleep, and high proportion of hypoxia and arousal events; therefore, he was diagnosed with severe obstructive sleep apnea.

By virtue of testing, patient believed that his airway was improved, and he did not snore when the mandible was repositioned to be advanced. At this point, an oral appliance was determined to be an appropriate treatment, and necessary records of the patient were obtained for the fabrication of the oral appliance.

A temporary mandible advancement device, called "NoSnore tray appliance", was selected for this patient (Fig. 1). This appliance was designed to test the effects of mandible advancement device (MAD) in patients with snoring and obstructive sleep apnea. ${ }^{4}$

The concept of this appliance is consistent with conventional "bite tray". This tray consisted of 2 parts as follows: the guide pin component that represents protrusion level of anterior teeth and connected custom tray component which allows free position of mandible. Polyvinylsiloxane impression material is mixed and filled into the tray, and the tray is then placed into the mouth of the patient while patient placed his incisor on pre-engraved position. The amount of mandible advancement is decided according to the incisal "edge to edge" bite position. When the impression material is hardened enough, the guide pin is removed and the appliance is trimmed. This fabrication process can be completed in a short time at the chair-side. The completed device is delivered to the patient, and it was instructed to be worn at bedtime every day.

During 2 months of using OA, the patient did not complain of any temporomandibular discomfort, while no change in his occlusion status was confirmed when patient attended the clinic after 2 months. The following PSG was proceeded to assess the effect of the treatment (Table 1). The results of the PSG revealed that the AHI was 2.4 and RDI was 5.9. His blood oxygen levels were in the $88.0 \%$ or greater range. The results showed a reasonable outcome with the oral appliance, and therefore, the temporary oral appliance was changed to a new full coverage appliance. 


\section{DISCUSSION}

For patients with severe OSA, the mostly recommended firstline treatment is CPAP device or surgical treatment, according to 2005 oral appliance treatment guideline. ${ }^{2}$ But, in this case, we tried an oral appliance treatment, without CPAP or surgical treatment, and there was an adequate improvement in severe sleep apnea (RDI 45.8). Hence, oral appliance may be considered as one of the first-line treatments for severe obstructive sleep apnea. However, additional clinical studies must be carried out, related to the conditions of each patient, to provide an appropriate treatment modality.

Temporary MAD can be fabricated at once by a dentist in clinic, with relatively low price compared to common mandible advancement devices. But, the extrusion of molar teeth may result from a long-term use of this appliance, as it only covers anterior teeth. So, a periodic occlusal examination is needed, and only a short-term application is recommended.

In conclusion, the temporary MAD can be considered, as it is simpler and inexpensive than common mandible advancement devices, for the patients even with severe OSA, as long as the protruded mandible position allows patient to breathe comfortably and decreases respiratory disturbance. Additionally, if the use of temporary MAD shows beneficial improvement in OSA, then the treatment plan can be corrected to a use of full coverage MAD or jaw advancement surgery.

\section{Acknowledgments}

This work was supported in part by the Yonsei University Future-leading Research Initiative of 2014 (2014-22-0131).

\section{Conflicts of Interest}

The authors have no financial conflicts of interest.

\section{REFERENCES}

1. Punjabi NM. The epidemiology of adult obstructive sleep apnea. Proc Am Thorac Soc 2008;5:136-43.

2. Kushida CA, Morgenthaler TI, Littner MR, Alessi CA, Bailey D, Coleman J Jr, et al. Practice parameters for the treatment of snoring and Obstructive Sleep Apnea with oral appliances: an update for 2005. Sleep 2006;29:240-3.

3. Clark GT, Sohn JW, Hong CN. Treating obstructive sleep apnea and snoring: assessment of an anterior mandibular positioning device. J Am Dent Assoc 2000;131:765-71.

4. Kim ST. Oral Appliance Therapy for Snoring \& OSA. 1st ed. Seoul: Myung Mun Publishing Co 2012. 